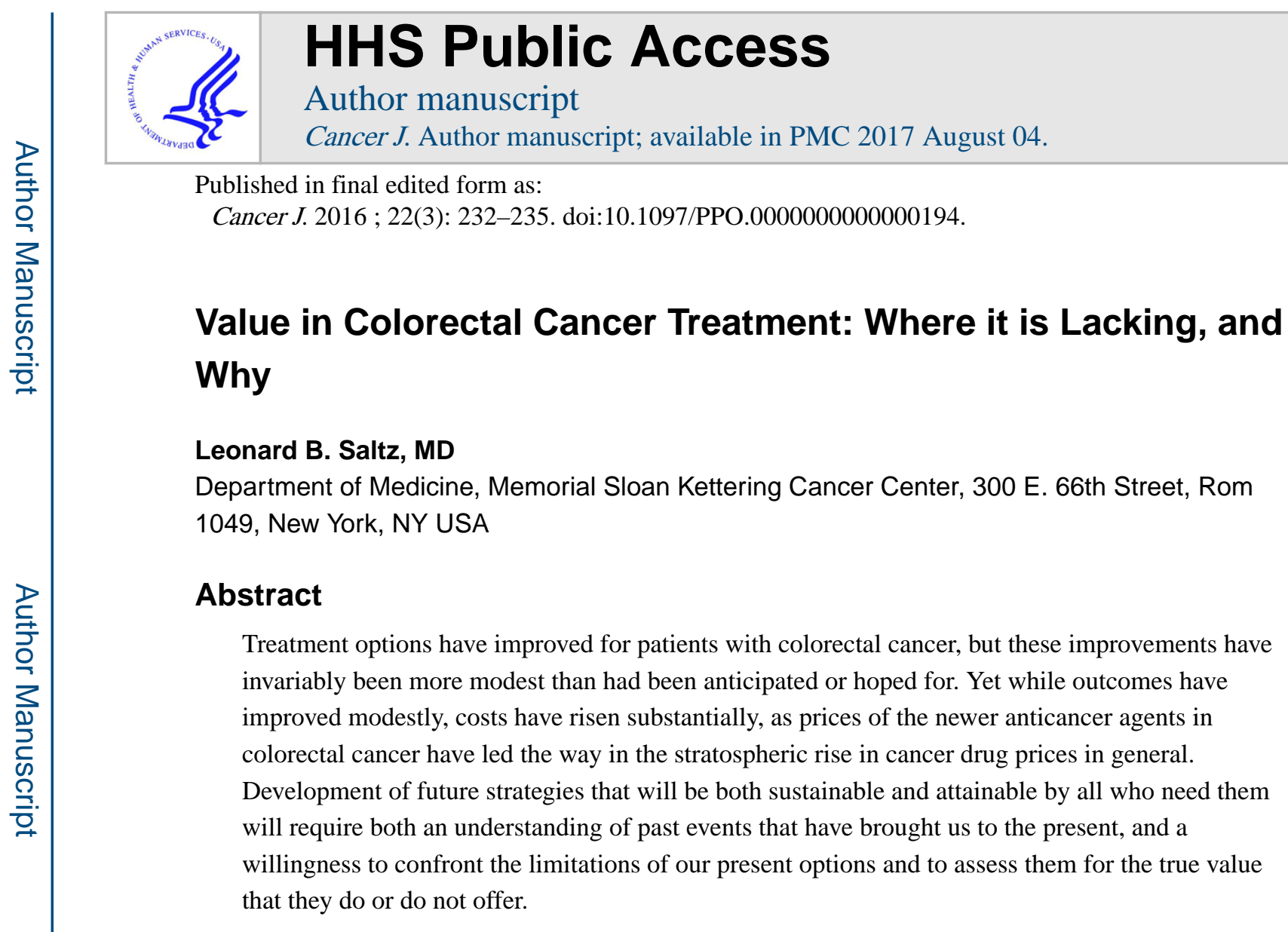

\title{
Introduction
}

As patients, their families, doctors, insurers, and policy makers have slowly become aware of the staggering cost of anticancer therapy and the meteoric rise in those costs, attention has begun to focus on those circumstances in which there is a profound disconnect between the cost of care and the value of the care that that cost is delivering. ${ }^{1}$ Nowhere is this dichotomy between cost and value more apparent than in gastrointestinal oncology in general, and in colorectal cancer (CRC) care in particular. Understanding how we got here and where we are now will be critical to establishing a future strategy that will be sustainable and deliverable to all of those who need it. Failure to confront the unsustainability of current practices, prices, and pricing trends now will invariably lead to even greater disparities in health care delivery than exist already, and such a course must not be allowed to be regarded as a viable option.

\section{In the Beginning}

For much of the second half of the twentieth century, as medical oncology began to emerge as a field, gastrointestinal (GI) malignancies remained stubbornly resistant to then-available therapeutic interventions, and GI malignancies came to be universally regarded as "chemorefractory." 5-Fluorouracil (5FU), an antimetabolite patented in August of 1957, was virtually the only agent available that demonstrated any meaningful antitumor activity, and

Correspondence to: Leonard B. Saltz, MD, Professor of Medicine, Weill Cornell Medical College, Chief, Gastrointestinal Oncology Service, Division of Solid Tumor Oncology, Department of Medicine, Memorial Sloan Kettering Cancer Center, 300 E. $66^{\text {th }}$ Street, Rom 1049, New York, NY USA, Tel: 646-888-4286, Fax: 646-888-4258, saltzl@ mskcc.org. 
its activity was accurately regarded as modest at best. In retrospect, the activity of $5 \mathrm{FU}$ was likely somewhat underestimated, in that diagnoses of GI malignancies were often made late, when patients already had large-volume disease and poor performance statuses, and so had a far less likely chance of either tolerating treatment or benefiting from it. Indeed, it remains unclear how much of the apparent progress seen in improved survival over the past several decades can be attributed to new drugs, versus how much is due to better use of surgery for metastatic disease, and better imaging leading to earlier awareness of the presence of metastatic cancer, thus creating a longer period of cancer diagnosis (lead time bias), but not necessarily as substantial a survival gain from the actual biologic onset of disease until death as has often been attributed to new drugs. ${ }^{2}$

Following the introduction of 5FU, GI oncology spent nearly the next forty years virtually wandering in a desert in terms of new drug development. It was not until 1996 that irinotecan became the second drug approved by the US FDA for use in CRC, and the brief "golden age" of CRC drug development began. Over the seven-year period from 1996 through 2003, data became available showing activity for irinotecan, oxaliplatin, capecitabine, bevacizumab, and cetuximab. Since that time, however, drug development in CRC has essentially stagnated, with only panitumumab, a drug nearly identical to cetuximab and so offering no real new clinical benefit, two additional inhibitors of the VEGF pathway, aflibercept and ramucirumab, each of which also confers no discernible benefit to patients over the already available alternative, and two oral agents, regorafenib and TAS-102, which each confer less than a two-month survival benefit, and that in highly selected group of chemotherapy-refractory patients.

\section{Progress, However Small, at Any Price}

Goldstein and El-Rayes have proposed a taxonomy of low-value care into two categories; type 1 low-value care (LVC) is defined as a new therapy that has a statistically significant but clinically modest benefit at a high cost, while type $2 \mathrm{LVC}$ is a treatment that adds no additional benefit in terms of efficacy or toxicity compared with the current standard care options and has a higher cost. ${ }^{3}$ As noted above, both types of LVC have entered into CRC management. ${ }^{4}$ How has this occurred? Because GI oncology from the 1960's through the early-mid 1990s was so bereft of legitimate progress, any and all activity of a novel antitumor agent was going to be gratefully embraced, as digestive tract cancers became the first of the solid tumor fields in which progress had been negligible, to show some glimmers of encouragement. As a cancer community, we were so happy to have something, anything, which "worked," by whatever definition we chose to use at the time, that we embraced each new agent with great enthusiasm, focusing on its benefits and diverting our attention from the reality that each drug that has followed 5FU was initially designed to exceed and replace $5 \mathrm{FU}$, and each has been unable to do so. The fallback position of drug development has been to then either combine that drug with 5FU in a combination regimen, or to sequence it in as a salvage therapy after $5 \mathrm{FU}$ failure. In that heady seven year period during which a new agent appeared on the scene seemingly every year and a half or so, there was a sense that the log jam had finally broken, and that although progress may have been slight, that this was just the beginning of what was to be an inexorable upward march towards highly efficacious and easily-tolerated treatments. Surely none of us thought that we had plateaued, or that the 
plateau we had reached was high enough. Yet, after nearly four decades of virtually no progress at all, a flurry of activity was perceived and received as both hopeful and exciting.

As new drugs entered the market, however, prices began to rise, and to rise in a manner dissociated from the amount of benefit delivered, as if these increases reflected more what the preclinical hopes for the drug had been, rather than what had actually be achieved in the clinic. ${ }^{5}$ Helping to drive the separation of price and value was the omnipresent notion that all that we do in medicine ought to be, and therefore is, above consideration of cost. As a society we have failed to recognize for some time that this notion is both impractical and unsustainable, and each time we try to confront this we fail to effectively do so. In that context, let's look at where we are in terms of treatments for CRC, what we are paying for it, and what we are getting for our money. Currently $5 \mathrm{FU}$, irinotecan, and oxaliplatin are off patent, and so relatively inexpensive generics are available, making these agents relatively minor contributors to drug costs. Whether this will remain the case or not remains to be seen. We have seen numerous situations in which an inexpensive generic drug, by accident or design, becomes limited in availability with only one supplier. Such circumstances have resulted in astronomical price increases, and current laws do not offer a remedy to this problem. ${ }^{6}$

Even so, recent practice, at least in the United States, indicates that oncologists have either ignored substantial price differences, or possibly have been induced by current reimbursement practices to favor the more expensive agents. An analysis of the C80405 NCI cooperative group trial in which the physicians were permitted to first make a choice between FOLFOX and FOLFIRI before patients were then randomized to receive either bevacizumab or cetuximab shows that physicians chose the oxaliplatin containing regimen approximately four times more often than the irinotecan-containing one, even though oxaliplatin at that time was still on patent and cost approximately ten times what generic irinotecan cost at the time. ${ }^{7,8}$ In addition, the primary results of the study showed essentially no clinical difference in outcome between use of cetuximab or bevacizumab in first line treatment of KRAS wild type CRC, and so no clinical preference was stated. This top line finding of the study was presented at the plenary session of the ASCO annual meeting in June of 2014. Segregated off in the health services outcomes presentations of the same meeting the following year, however, was an analysis showing that the cost of cetuximab was more than double that of bevacizumab. ${ }^{9}$ This should not lead us to the conclusion that either cetuximab or bevacizumab is an equally acceptable alternative, rather that bevacizumab, despite its already high cost ${ }^{10}$ is the clear favorite on the basis of lower-cost, barring a particular extenuating circumstances in terms of relative risk of toxicity profile. In short, it is not that no one should get cetuximab up front, but rather that no one should unless there is a particular reason why they should not receive bevacizumab instead.

Unfortunately, the current "buy and bill" method of drug reimbursement, in which the percentage that the purchasing doctor or hospital is paid is tied to the cost of the drug, creates the undesirable but also undeniable incentive for the purchasing individual or organization to use the most expensive regimen possible. While each of us would like to believe that we were above such temptations, it would be difficult to ignore the likelihood 
that an individual, perceiving no real medical difference to his or her patient, would not then be inclined to choose that course of action which is felt to be most beneficial to the provider.

\section{Falling into the VEGF Trap}

Perhaps in no place has the disconnect between value and benefit been more apparent than in the introduction of newer anti-VEGF agents into the treatment of CRC. Aflibercept, an elegantly engineered molecule designed to bind all isoforms of VEGF, was hoped to be a superior agent to the already available bevacizumab, an anti-VEGF monoclonal antibody. That this was unlikely to be the case became apparent early on in the clinical development of this agent, however, leading the developers to assiduously avoid any head-to-head comparisons with bevacizumab. They chose instead to study aflibercept in combination with FOLFIRI in second line CRC, where it showed a median improvement in overall survival of 1.4 months when added to FOLFIRI over FOLFIRI alone. ${ }^{11}$ At the same time, however, a study evaluating continuation of bevacizumab beyond progression had been initiated by the AIO German cooperative group. The manufacturer of bevacizumab acquired that study in progress and substantially increased it in size. The results strikingly paralleled those of the aflibercept study, with again a 1.4 month median survival benefit for use of bevacizumab with second line therapy after its use in first line therapy as well. ${ }^{12}$ It is noteworthy that had the trial remained with the AIO at the originally intended size, it is a very real possibility that the difference in survival would not have achieved statistical significance. It is further noteworthy that a cost effectiveness analysis of bevacizumab in both first and second line treatment of CRC found it to fail to meet any typically accepted standards of cost effectiveness. ${ }^{13}$ Nevertheless, when aflibercept gained FDA approval, it was placed on the market at a cost that was more than double that of bevacizumab. Comments in the media from executives involved in the marketing decisions for aflibercept noted that their price was targeted towards the $10 \mathrm{mg} / \mathrm{kg}$ dose of bevacizumab, a dose which is not listed in NCCN guidelines for any phase of CRC treatment, and which was rejected by the NCI Colon Cancer Task Force as inappropriate for study in CRC in the adjuvant setting. That the developers of aflibercept were able to locate some practitioners who were using the 10 $\mathrm{mg} / \mathrm{kg}$ dose is concerning, as in the opinion of this author the use of $10 \mathrm{mg} / \mathrm{kg}$ of bevacizumab in CRC is not appropriate, and reflects either a lack of understanding of the literature at best, or cynical avariciousness at worst.

At Memorial Sloan Kettering Cancer Center in New York, upon being made aware of these cost issues, the doctors who treat CRC at that institution agreed that they did not need aflibercept to provide optimal care to their patients and so did not plan to use it. The drug was therefore not added to the hospital formulary. This decision, representing the first time to our knowledge that a new anticancer agent for a common malignancy was deliberately not added to the formulary of a major cancer center on the basis of cost, was published as an opinion piece in the mainstream media. ${ }^{14}$ Within a month of that publication, the manufacturer offered a 50\% discount in the cost of the drug to all oncologists in the United States, ${ }^{15}$ showing the inherent liquidity in prices and the ability of market forces, when allowed to be brought into play, to impact on those prices. 
Unfortunately, we who do not learn from history are condemned to repeat it. In 2015, the FDA approved the use of the VEGF receptor inhibitor ramucirumab in conjunction with FOLFIRI in second line treatment of CRC. The study design looked remarkably like that for aflibercept, and the results looked remarkably the same, with an extremely modest improvement in overall survival comparable to that achievable with either aflibercept or bevacizumab. ${ }^{16}$ By this time, however, ramucirumab was already on the market at more than two and a half times the price of $5 \mathrm{mg} / \mathrm{kg}$ dose of bevacizumab for use in other indications in which bevacizumab was not registered. ${ }^{3}$ Again the $10 \mathrm{mg} / \mathrm{kg}$ bevacizumab dose appears to have been the benchmark for pricing. As seen in table 1, this agent provides no meaningful benefit over the other two anti-VEGF agents. Furthermore, no data exist and no good hypothesis exists to suggest that use of one of these agents after failure of another will be beneficial. Use of ramucirumab in patients with $\mathrm{CRC}$ at this time is therefore inappropriate, given that at current prices it is more than twice the cost of bevacizumab, with no indication that it offers either a therapeutic advantage or an improved toxicity profile.

\section{Small Molecules with Small Benefits and Large Price Tags}

In the last several years two oral agents, regorafenib and TAS-102, have received FDA approval for use in patients with chemotherapy-refractory CRC. Each of these agents was developed in a highly selected population of patients who still had excellent performance status and good bone marrow, liver, and kidney function after progression through all available standard therapies. Even in these selected individuals, the incremental survival benefits of these agents were 1.4 and 1.8 months respectively with toxicity that was far from trivial. ${ }^{17,18}$ These agents entered the market in the general range of $\$ 10,000$ to $\$ 12,000$ per patient per month, the going rate for new anticancer agents at the time, regardless of the incremental degree of benefit they offered. ${ }^{19}$ These costs, well exceeding $\$ 100,000$ per patient per year, are striking both in that they offer only minimal benefit, with incremental cost effectiveness ratios that do not meet standards for cost effectiveness, ${ }^{20}$ but bring home to patients the issue of direct financial toxicity, in that they are specialty pharmacy drugs which can carry patient co-pays as high as $20 \%$.

\section{Immunotherapy and the big step forward for a very small number}

The journal Science designated immunotherapy for cancer as the most important scientific advance of the year 2013. Drugs working on a mechanism of immune checkpoint inhibition have substantially changed the landscape for therapy of melanoma, and have made meaningful progress in the care of patients with cancers of the kidney, lung, and those with lymphomas, amongst others. Progress in CRC, however, has been disappointing. The CTLA4 inhibitor tremelimumab was studied in CRC a decade ago, however the results were disappointing. ${ }^{21}$ Studies with PD1 inhibitors were also disappointing, with the exception of the rare subgroup of metastatic CRC patients who have both metastatic disease and tumors characterized by mismatch repair protein deficiency. To date, one prominent report has shown an encouraging response rate with some degree of durable disease control in patients with mismatch repair deficient CRC, while finding essentially no activity in mismatch repair proficient, or microsatellite stable, CRC. ${ }^{22}$ Owing to the fact that these are favorable prognosis tumors, while approximately $15 \%$ of CRCs are mismatch repair deficient, the vast 
majority of these never go on to develop metastatic disease, but rather present in stages 1, 2, or 3. Only a few percent (estimates of $1 \%-4 \%$ ) of patients who develop metastatic CRC have mismatch repair deficiency disease. For these people the PD1 inhibitor pembrolizumab has been shown to have activity. However the FDA-approved dose of this drug is $2 \mathrm{mg} / \mathrm{kg}$ every 3 weeks. The study that reported the efficacy of this drug in mismatch repair deficiency CRC, however, utilized five times that dose, or $10 \mathrm{mg} / \mathrm{kg}$. No studies have demonstrated that a dose higher than $2 \mathrm{mg} / \mathrm{kg}$ every 3 weeks has greater efficacy. It is notable that at current prices a dose of $10 \mathrm{mg} / \mathrm{kg}$ would, for a $75 \mathrm{~kg}$ individual, exceed an annual price tag of $\$ 1$ million per patient per year.

\section{This is where we are. Where will we go next?}

Ultimately our problem in establishing value in CRC, as well as just about any other cancer, stems from a deep discomfort with confronting the question of just what is a given amount of benefit worth? What are we willing to pay, financially, for an extension of life by a month, or a year? Is a drug that extends life by a year worth twelve times that of one which extends life by a month? If so, is the one that extends life by a month only worth one twelfth the price of one which extends life by a year? As uncomfortable as these questions are, we must confront them or we will make no progress in getting out of the financial quagmire that we are in. At present, although we choose not to notice, we are routinely accepting a price of at least $\$ 300,000$ per year of life saved. This is the cost, for example of a year of life saved by adding bevacizumab or aflibercept to second line FOLFIRI. Yet if we were to use second line ramucirumab, that cost would more than double, and yet we permit such an option to be available. Clearly we have failed as a society to confront these inconsistencies between high cost and low benefit. It is a luxury we never really could afford. It is becoming clearer and clearer that we cannot afford it now. How will we deal with this? Will we muster the collective nerve and sense of responsibility to walk away from an overpriced minimal benefit, because we simply cannot and should not afford it? If the answer is no, then we are forever constrained to have more and more small benefits at higher and higher cost. Only through abandoning the practice of consistently using low value care (and paying high prices for it) will we be able to move towards high-value care for our patients. If we refused to pay a high price for low value care, then market forces will bring that price down. Until we do so, the field of CRC care will continue to be the veritable poster children for high dollar, minimal benefit, and therefore low value, care.

\section{References}

1. Saltz LB. The Value of Considering Cost, and the Cost of Not Considering Value. Journal of Clinical Oncology. 2016; 34:659-60. [PubMed: 26668345]

2. Jawed I, Wilkerson J, Prasad V, Duffy AG, Fojo T. Colorectal cancer survival gains and novel treatment regimens: A systematic review and analysis. JAMA Oncology. 2015; 1:787-95. [PubMed: 26181239]

3. Goldstein DA, El-Rayes BF. Considering Efficacy and Cost, Where Does Ramucirumab Fit in the Management of Metastatic Colorectal Cancer? The Oncologist. 2015; 20:981-2. [PubMed: 26265225]

4. Goldstein DA, Zeichner SB, Bartnik CM, Neustadter E, Flowers CR. Metastatic Colorectal Cancer: A Systematic Review of the Value of Current Therapies. Clinical Colorectal Cancer. 2016; 15:1-6. [PubMed: 26541320] 
5. Saltz LB. Perspectives on cost and value in cancer care. JAMA Oncology. 2016; 2:19-21. [PubMed: 26501848]

6. Alpern JD, Stauffer WM, Kesselheim AS. High-Cost Generic Drugs - Implications for Patients and Policymakers. New England Journal of Medicine. 2014; 371:1859-62. [PubMed: 25390739]

7. Venook APND, Lenz H-J, et al. CALGB/SWOG 80405: Phase III trial of irinotecan/5-FU/ leucovorin (FOLFIRI) or oxaliplatin/5-FU/leucovorin (mFOLFOX6) with bevacizumab (BV) or cetuximab (CET) for patients (pts) with KRAS wild-type (wt) untreated metastatic adenocarcinoma of the colon or rectum (MCRC). J Clin Oncol. 2014; 32(suppl; abstr LBA3)

8. Saltz LB. The cost and value of anti-epidermal growth factor receptor therapies: Let's not be rash. JAMA Oncology. 2015; 1:141-2. [PubMed: 26181010]

9. Schrag DD,AC, Naughton MJ. Cost of chemotherapy for metastatic colorectal cancer with either bevacizumab or cetuximab: Economic analysis of CALGB/SWOG 80405. J Clin Oncol. 2015; 33(15_suppl):6504. Meeting Abstracts.

10. Saltz LB. Can Money Really Be No Object When Cancer Care Is the Subject? Journal of Clinical Oncology. 2015; 33:1093-4. [PubMed: 25691668]

11. Van Cutsem E, Tabernero J, Lakomy R, et al. Addition of Aflibercept to Fluorouracil, Leucovorin, and Irinotecan Improves Survival in a Phase III Randomized Trial in Patients With Metastatic Colorectal Cancer Previously Treated With an Oxaliplatin-Based Regimen. Journal of Clinical Oncology. 2012; 30:3499-506. [PubMed: 22949147]

12. Bennouna J, Sastre J, Arnold D, et al. Continuation of bevacizumab after first progression in metastatic colorectal cancer (ML18147): a randomised phase 3 trial. The Lancet Oncology. 2013; 14:29-37. [PubMed: 23168366]

13. Goldstein DA, Chen Q, Ayer T, et al. First- and Second-Line Bevacizumab in Addition to Chemotherapy for Metastatic Colorectal Cancer: A United States-Based Cost-Effectiveness Analysis. Journal of Clinical Oncology. 2015; 33:1112-8. [PubMed: 25691669]

14. Bach PB, Wittes RE, Saltz LB. Cancer Care, Cost Matters. New York Times. 2012 Oct 14.

15. Pollack A. Sanofi Halves Price of Cancer Drug Zaltrap After Sloan-Kettering Rejection. New York Times 2012 November 8. 2012

16. Tabernero J, Yoshino T, Cohn AL, et al. Ramucirumab versus placebo in combination with secondline FOLFIRI in patients with metastatic colorectal carcinoma that progressed during or after firstline therapy with bevacizumab, oxaliplatin, and a fluoropyrimidine (RAISE): a randomised, double-blind, multicentre, phase 3 study. The Lancet Oncology. 2015; 16:499-508. [PubMed: 25877855]

17. Grothey A, Cutsem EV, Sobrero A, et al. Regorafenib monotherapy for previously treated metastatic colorectal cancer (CORRECT): an international, multicentre, randomised, placebocontrolled, phase 3 trial. The Lancet. 2013; 381:303-12.

18. Mayer RJ, Van Cutsem E, Falcone A, et al. Randomized Trial of TAS-102 for Refractory Metastatic Colorectal Cancer. New England Journal of Medicine. 2015; 372:1909-19. [PubMed: 25970050]

19. Mailankody S, Prasad V. Five years of cancer drug approvals: Innovation, efficacy, and costs. JAMA Oncology. 2015; 1:539-40. [PubMed: 26181265]

20. Goldstein DA, Ahmad BB, Chen Q, et al. Cost-Effectiveness Analysis of Regorafenib for Metastatic Colorectal Cancer. Journal of Clinical Oncology. 2015; 33:3727-32. [PubMed: 26304904]

21. Chung KY, Gore I, Fong L, et al. Phase II Study of the Anti-Cytotoxic T-Lymphocyte-Associated Antigen 4 Monoclonal Antibody, Tremelimumab, in Patients With Refractory Metastatic Colorectal Cancer. Journal of Clinical Oncology. 2010; 28:3485-90. [PubMed: 20498386]

22. Le DT, Uram JN, Wang H, et al. PD-1 Blockade in Tumors with Mismatch-Repair Deficiency. New England Journal of Medicine. 2015; 372:2509-20. [PubMed: 26028255] 


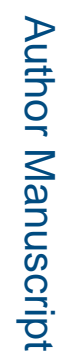

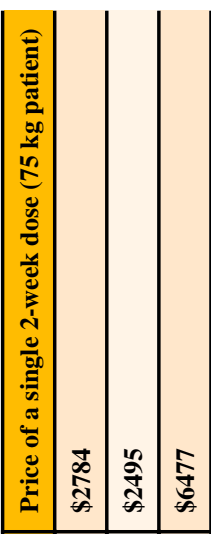

要

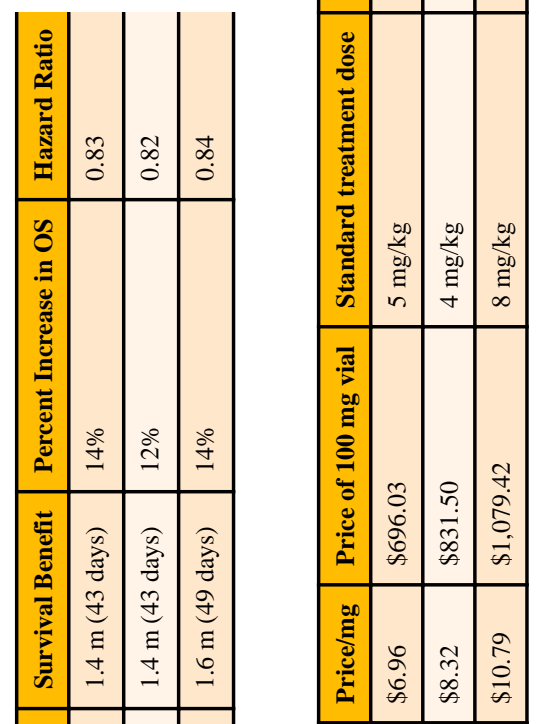

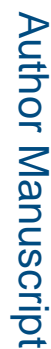
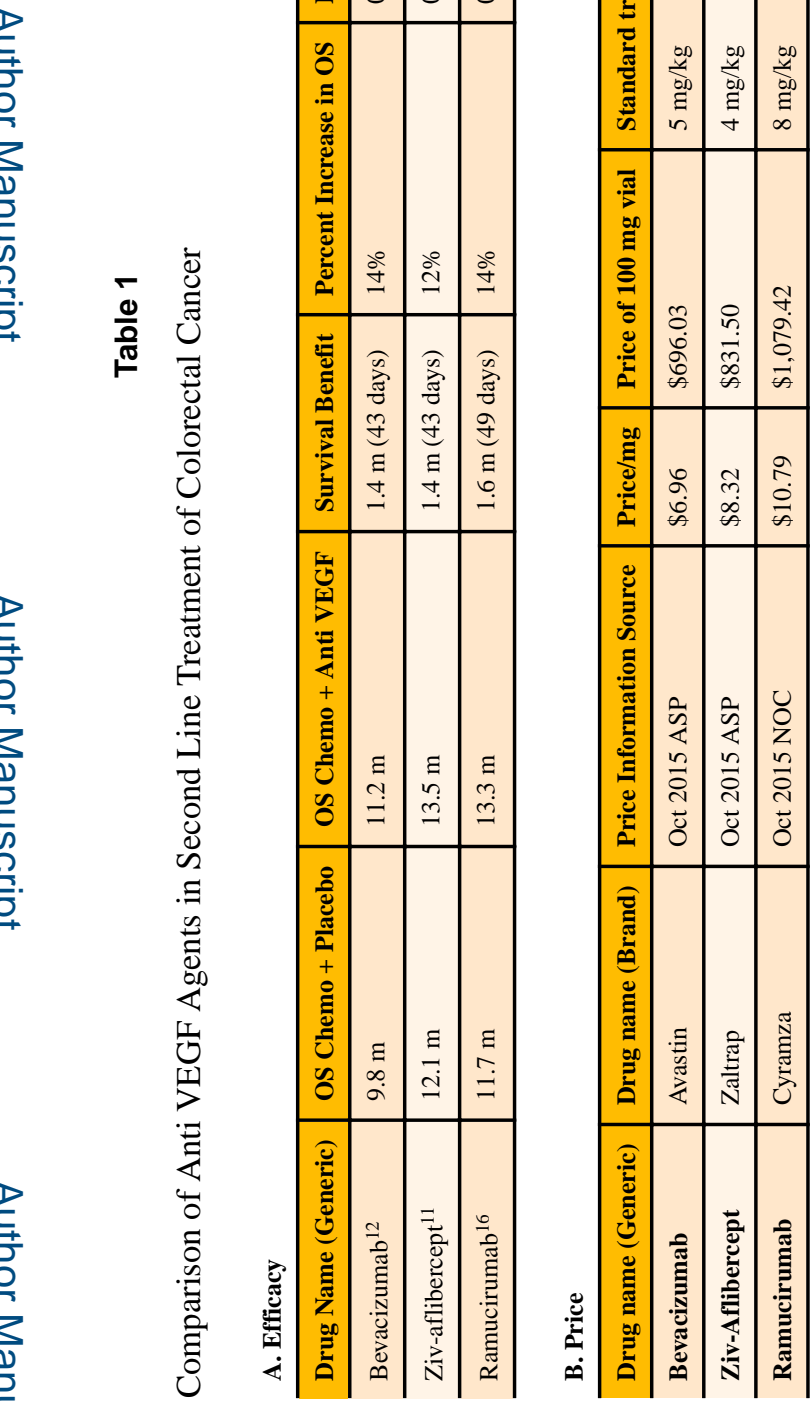

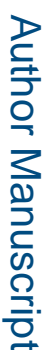

